\title{
A new cost-effective staining method for rapid identification of Cryptococcus
}

\author{
Rugen Wan, Maofeng Wang ${ }^{*}$, Sheng Zhao, Guogang Li, Yangxiao Zhou, \\ Jiajun Li and Jinkan Du
}

Department of Clinical Laboratory, Affiliated Dongyang Hospital (Dongyang People's Hospital) of Wenzhou Medical College, Dongyang, Zhejiang, 322100, P. R. China.

Accepted 23 June, 2011

\begin{abstract}
Cryptococcosis is a life-threatening fungal disease that usually presents as an opportunist infection in immunocompromised hosts, and Cryptococcus neoformans is the major opportunistic pathogen of cryptococcosis. Rapid diagnosis of $\boldsymbol{C}$. neoformans and other opportunistic Cryptococcus pathogens is required to meet the clinical need for immediate treatment. Historically, negative staining with India ink has been used to identify Cryptococcus. Another staining method utilizes Alcian blue, a dye that reacts selectively with $C$. neoformans, but not other Cryptococcus species. To overcome these limitations, we developed a new staining method for rapid identification of Cryptococcus using a novel, patent pending staining reagent for clinical laboratory diagnosis. Cryptococci stained with this new reagent can be conveniently counted directly via bench-top light microscopy. We also compared our novel stain with India ink and Alcian blue. Our results demonstrated that the new staining technique was superior to India ink stain for the rapid identification of Cryptococcus in clinical (and clinically mimicking) samples. Our stable and cost-effective staining reagent specifically stained Cryptococcus, highlighting its clinical applicability, particularly in developing countries due to its low cost.
\end{abstract}

Key words: Cryptococcus, cryptococcosis, specific staining, India ink staining, rapid identification.

\section{INTRODUCTION}

Cryptococcosis or cryptococcal disease is a potentially fatal fungal disease that occurs in immunocompromised hosts due to an opportunist infection (Perfect and Casadevall, 2002; Gazzoni et al., 2009). Cryptococcus neoformans and, somewhat less commonly, Cryptococcus gattii are the major opportunistic pathogens of this life-threatening disease as well as other human infections, including pulmonary infections and meningitis (Harding et al., 1979). Cryptococcus species other than C. neoformans have long been generally regarded as nonpathogenic saprophytes. However, other Cryptococcus fungi species (example, Cryptococcus albidus, Cryptococcus curvatus, Cryptococcus humicolus,

${ }^{\star}$ Corresponding author. E-mail: wzmcwmf@163.com. Tel: +86139-6799-5216. Fax: +86-579-8685-6878.
Cryptococcus uniguttulatus, and Cryptococcus laurentii) have also been associated with opportunistic infections (Kamalam et al., 1977; Velez et al., 1996; Johnson et al., 1998; Kordossis et al., 1998; Ritterband et al., 1998; McCurdy and Morrow, 2001).

Due to the wide spectrum of cryptococcosis and their opportunistic nature, rapid laboratory identification of Cryptococcus pathogens is needed to enable initiation of therapy without delay. Thus, in recent years, the need for rapid and accurate identification of Cryptococcus in the clinical laboratory setting has led to the development of many identification assays. Laboratory diagnosis of cryptococcosis includes conventional methods (example, direct microscopic examination and histopathology) (Gal et al., 1986; Perfect and Casadevall, 2002; Chayakulkeeree and Perfect, 2006; Gazzoni et al., 2008; 2009) that are usually associated with serology (Bloomfield et al., 1963; Berlin and Pincus, 1989; Leal et 
al., 2008) and isolation of the offending organism in culture (Berlin and Pincus, 1989; Perfect and Casadevall, 2002; Bovers et al., 2008).

While a final diagnosis can ultimately be confirmed through a culture of the organism, the urgency to initiate treatment quickly requires that a presumptive diagnosis be made by direct examination of cerebrospinal fluid (CSF) and blood cultures. India ink stain testing has historically been used for this purpose, but India ink staining yields a positive result in only $\sim 60 \%$ of cases of culture-proven infection (Butler et al., 1964).

Microscopically, Cryptococcus yeast cells are spherical-to-oval in shape, 5 to $10 \mathrm{~mm}$ in diameter, and are surrounded by a polysaccharide capsule (Baker and Haugen, 1955), which constitutes a major virulence factor and the substrate detected by cryptococcal antigen tests (CrAg) (Bloomfield et al., 1963). However, capsuledeficient Cryptococcus infections may result in falsenegative results using this test (Harding et al., 1979; Monteil et al., 1997; Gazzoni et al., 2009). The rounded, large, "yeast-like" Cryptococcus cells can be counted directly with a blood cell counter.

However, it can be difficult to distinguish Cryptococcus cells from impurities, particulates, and white blood cells in unstained clinical samples (example, CSF). The Alcian blue stain method can also be used in the diagnosis of some types of cryptococcosis, such as salivary cryptococcosis in AIDS patients with disseminated $C$. neoformans infections (Monteil et al., 1997). However, the Alcian blue method has very limited application in detection of other Cryptococcus fungi because the stain only reacts with the $C$. neoformans capsule (Lazcano et al., 1993).

Here, we describe a new staining method for the rapid identification of Cryptococcus using a novel, patent pending staining reagent, namely Wan's staining reagent, which contains the commonly-used Evans blue dye. Cryptococcus staining with this cost-effective staining reagent enables convenient direct cell counting under a bench-top light microscope. We compared the staining results obtained using Wan's staining reagents relative to India ink and Alcian blue staining.

\section{MATERIALS AND METHODS}

\section{Staining reagents}

Wan's staining reagent, named after its inventor Dr. Ru-Gen Wan (the primary author of this article), was made by thoroughly mixing $1.0 \mathrm{~g}$ Evans blue dye (Direct Blue 53, Fluka, Switzerland) in a solution of $1.0 \mathrm{ml}$ glacial acetic acid and $99.0 \mathrm{ml}$ distilled water. The cost for each of these constituents was negligible. A complete Wan's staining reagent recipe has been filed for an invention patent in China (patent pending, application number: 200910154521.1). A $10 \mathrm{~g} / \mathrm{L}$ working solution of Alcian blue staining reagent was made by thoroughly mixing $1.0 \mathrm{~g}$ Alcian blue (Chroma, Germany) in a solution of $1.0 \mathrm{ml}$ glacial acetic acid and $99 \mathrm{ml}$ distilled water. Ready-to-use India ink was purchased from Yidege Industry and Trade Center (Beijing, China).

\section{Fungal strains and media}

C. neoformans, Candida albicans, Candida tropicalis, Candida glabrata, Candida krusei, Candida guilliermindii, Candida parapsilosis, and Saccharomyces cerevisiae clinical isolates from human patients were used. The identity of each strain was confirmed by ATB expression system kits (bioMérieux Vitek, France), which are commonly used for microbial identification in clinical microbiology laboratories. The use of human samples was approved by the Wenzhou Medical College Human Investigation Committee. C. albidus, C. laurentii, Candida lusitaniae and Candida rugosa obtained from the Clinical Laboratory Center of Zhejiang Province were used as provincial external quality control strains. Two quality control Cryptococcus strains, C. neoformans (ATCC 32609) and C. laurentii (ATCC 18803), were kind gifts from the Clinical Laboratory Center of Zhejiang Province. All strains were maintained on Sabouraud's agar slants and subcultured at $35^{\circ} \mathrm{C}$ for 24 to $48 \mathrm{~h}$ before each experiment.

\section{Instruments}

Specimens were examined using an Olympus micro-diagnosis system (Olympus BX 51, Japan).

\section{Specificity and stability of Wan's staining reagent}

Sample preparation A was produced by mixing human white blood cells with Cryptococcus and Candida in $2 \mathrm{ml}$ physiological saline to obtain suspended samples. Sample preparation B was produced by individually mixing Cryptococcus with each of the aforementioned fungi strains to obtain mixed samples. The above-described samples were each stained with Wan's staining reagent by mixing $50 \mu \mathrm{l}$ of each sample with $50 \mu \mathrm{l}$ of stain solution.

For microscopic observation, the stained samples were placed on slides, covered with a cover slip, examined under 5 dca microscope with a $40 \times$ and $100 \times$ objective, and then examined further under an oil immersion $(100 x)$ objective to evaluate the specificity of Wan's staining reagent.

To test the stability of Wan's staining reagent, the staining results obtained using Wan's staining reagent stored at room temperature for 1 year, 6 months, or 1 month or freshly made on the day of testing were assessed. To assess the staining stability of the Wan's staining method, staining was evaluated after various durations (1, 5,30 , and $60 \mathrm{~min}$ ) of staining.

\section{Clinical application of Wan's staining reagent}

A total of 73 CSF specimens collected from encephalitis patients, 11 lavage fluid specimens collected from patients with a lower respiratory infection, and 3 blood cultures were used in this study. These clinical samples were stained with either Wan's staining reagent or India ink to identify Cryptococcus fungi. If Cryptococcus was detected in the clinical samples, the fungal cells were counted manually using a blood cell counter. Cultured Cryptococcus aliquots from CSF were diluted to three different concentration ranges (10 to 20,100 to 200 , and 1000 to 2000 cells/ $\mu$ l) to obtain 10 samples that mimic clinical samples. These 10 samples were then coded and examined by 10 laboratory technicians (three senior, four mid-level, and three junior technicians) blinded to the sample identities. Three replicates of each sample were stained with Wan's staining reagent, Alcian blue, and India ink and then examined. We then counted the numbers of stained cells using a blood cell counter and compared the counting precision of Cryptococcal cells stained with Wan's reagent versus cells stained with Alcian blue. 


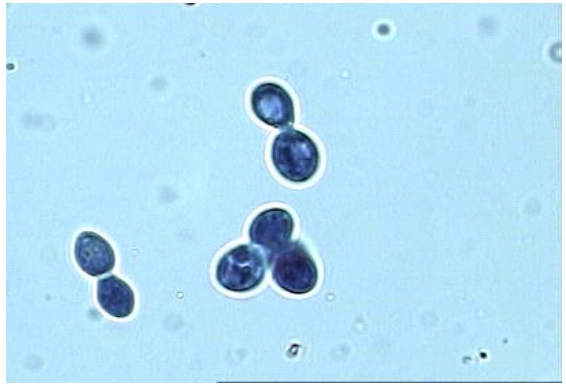

A

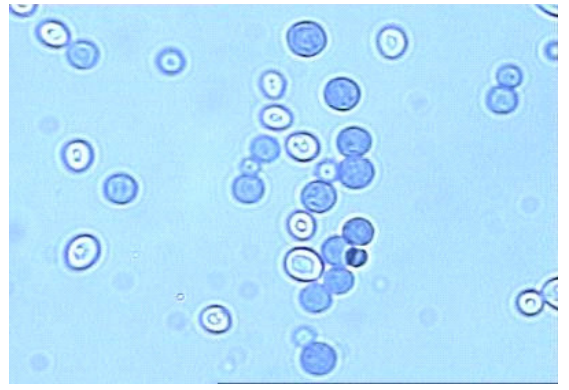

B

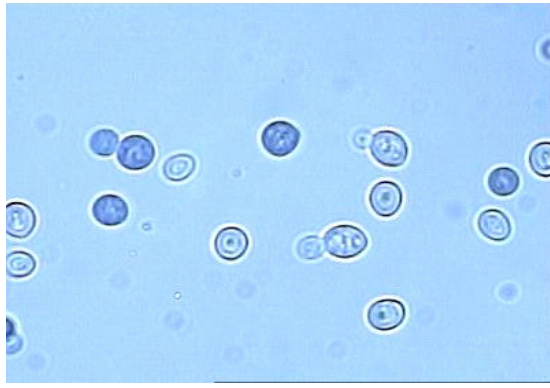

C

Figure 1. Staining results for common fungi with Wan's reagent (40x objective). A: Cryptococcus albidus cells were stained blue with Wan's reagent. B: Quality control strain cells of Cryptococcus neoformans (ATCC 32609) were stained blue, but Candida glabrata cells were unstained. C: Quality control strain cells of Cryptococcus laurentii (ATCC 18803) were stained blue, whereas Candida species (C. tropicalis and $C$. glabrata) were unstained.

\section{RESULTS}

\section{Staining clinically common fungi with Wan's staining reagent}

Clinically common Cryptococcus species or mixtures of Cryptococcus and other clinically common Candida species were simultaneously stained with Wan's staining reagent to investigate the reagent's specificity. Typical staining results are shown in Figure 1. C. albidus (Figure $1 \mathrm{~A}$ ) and $C$. laurentii (not shown) from clinical isolates and quality control $C$. neoformans (Figure 1B) and $C$. laurentii (Figure 1C) strains were stained blue or dark blue, while white blood cells and common Candida fungi [example, $C$ glabrata (Figure 1B), C. tropicalis (Figure 1C)] were unstained. It should be noted that $C$. rugosa fungi were also stained, but the staining affected $<1 \%$ of these cells. Therefore, stained cryptococcal cells were clearly distinguishable from clinically common Candida fungi, highlighting the high specificity of Wan's staining reagent for cryptococcal fungi.

\section{Staining stability of Wan's staining reagent}

The staining stability of Wan's staining reagent for mixtures of Cryptococcus and Candida fungi was compared after various durations $(1,5,30$, and $60 \mathrm{~min})$ of staining. Cryptococcus fungi were stained in as quickly as $1 \mathrm{~min}$ with Wan's staining reagent, while Candida fungi remained unstained (Figure 2A). Wan's staining reagent demonstrated high-quality staining stability as excellent staining results were maintained for up to $1 \mathrm{~h}$ of staining (Figures 2B to D).

\section{Stability of Wan's staining reagent}

Wan's staining reagent proved to be very stable as stained cryptococcal cells were still clearly distinguishable from Candida fungi after simultaneously staining with Wan's stain that had been stored at room temperature for up to 1 year (Figures $3 A$ to $D$ ).

\section{Clinical application of Wan's staining reagent}

We further evaluated the potential clinical application of Wan's reagent for the rapid identification of Cryptococcus in clinical samples. As a comparison, the traditional India ink staining method was used to stain clinical specimens or samples mimicking clinical specimens. CSF, lavage fluid, and blood culture samples were stained with Wan's staining reagent or India ink to identify Cryptococcus fungi. Cryptococcal cells were detected in $3 / 73 \mathrm{CSF}$ specimens and $3 / 3$ blood culture samples. No Cryptococcus fungi were identified in any of the 11 lavage fluid specimens.

Like Wan's staining reagent, Alcian blue also stained Cryptococcus fungi. However, Alcian blue often also caused aggregation of the cryptococcal cells, particularly when handled by less experienced laboratory technicians. A high background of India ink staining could make false negative staining a problem when only a limited amount of Cryptococcus fungal cells with a small capsular size are present. In contrast, cryptococcal cells stained with Wan's reagent appeared blue with bright capsules on a clear background without any aggregation. Typical staining results for clinical samples using the three staining methods are shown in Figures 4A to $\mathrm{C}$.

When we compared the false negative rates of our new staining method with India ink staining for the 10 mock clinical samples, we observed a lower false negative rate for Wan's reagent than for India ink staining. Indeed, only three false negatives (3/100) occurred with Wan's reagent, and these occurred exclusively at low concentrations of cryptococcal cells (10 to 20 cells $/ \mu \mathrm{l}$ ). Conventional India ink staining resulted in a higher 

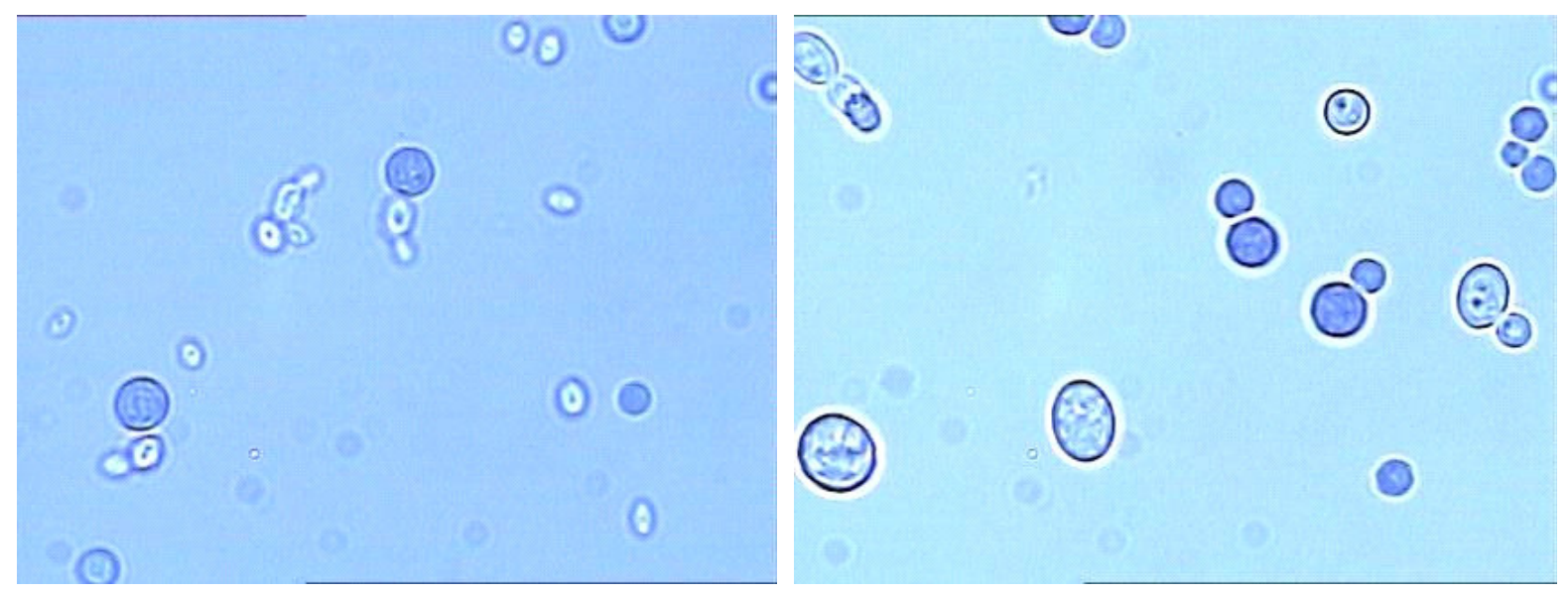

A

B

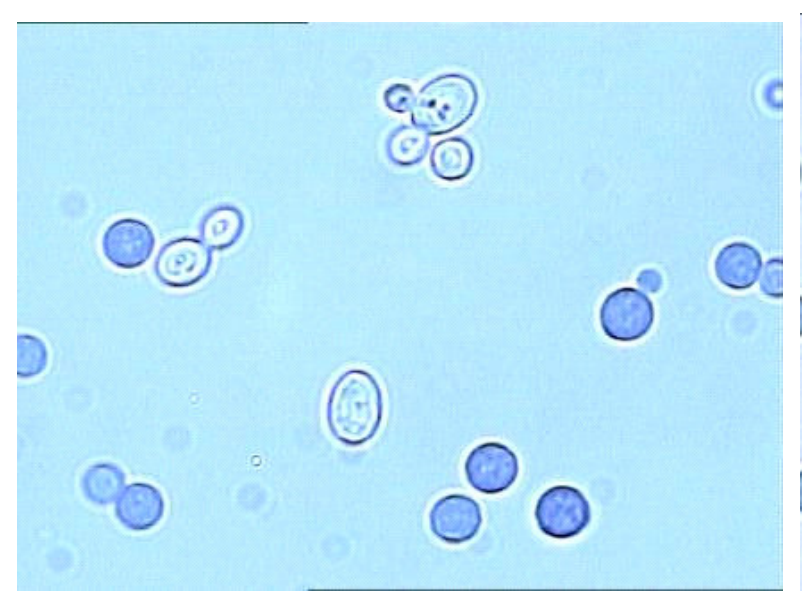

C

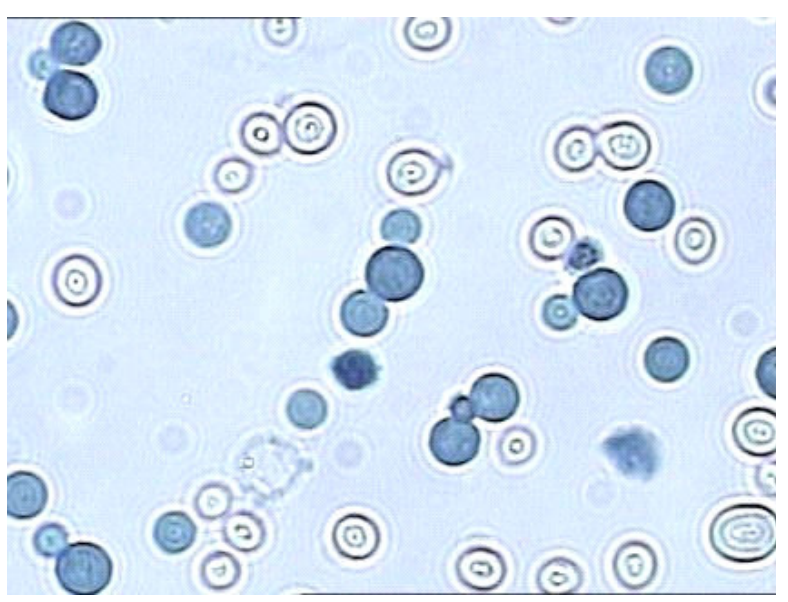

D

Figure 2. Staining stability of Wan's reagent. The staining stability of Wan's reagent on a mixture of Cryptococcus and Candida fungi was compared after various periods of staining: $1 \mathrm{~min}(\mathrm{~A}), 5 \mathrm{~min}$ (B), $30 \mathrm{~min}$ (C), and $60 \mathrm{~min}$ (D). Cryptococcus cell staining was apparent in as quickly as 1 min with Wan's staining reagent, whereas Candida cells remained unstained even after $60 \mathrm{~min}$.

frequency of false negatives (10/100), including eight false negative results at low concentrations of cryptococcal cells (10 to 20 cells $/ \mu \mathrm{l}$ ) and two at a medium concentration (100 to 200 cells $/ \mu$ l).

\section{Counting precision of cryptococcal cells stained with Wan's reagent}

As reported earlier, cryptococcal cells staining Wan's reagent were clearly distinguishable from white blood cells and Candida fungi. The clear background observed with Wan's stain allowed unproblematic and efficient counting of cryptococcal cells against the clear grid lines of the blood cell counter (Figure 5). In contrast, the cell aggregation caused by Alcian blue staining made precision counting difficult. Thus, better precision was obtained with Wan's reagent than Alcian blue stain in mock clinical samples of cryptococcal cells at three cell concentrations $\left(2 \times 10^{3}, 2 \times 10^{2}\right.$, and $\left.2 \times 10 \mathrm{cells} / \mathrm{\mu l}\right)$. Lower mean cell count values were consistently reported with Alcian blue stain due to the higher false negative rate of Alcian blue staining (described earlier). The cell count results are summarized in Table 1. 


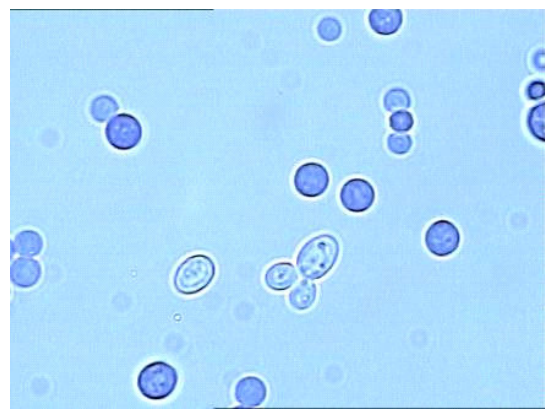

A

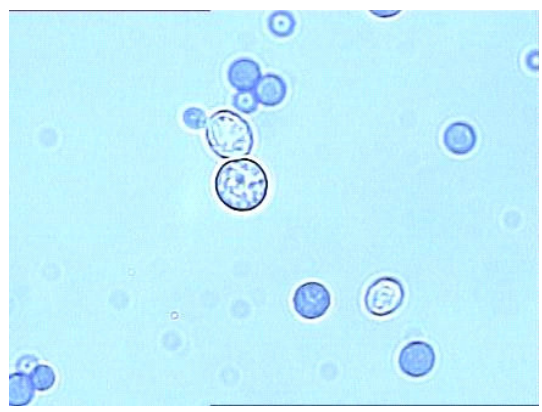

C

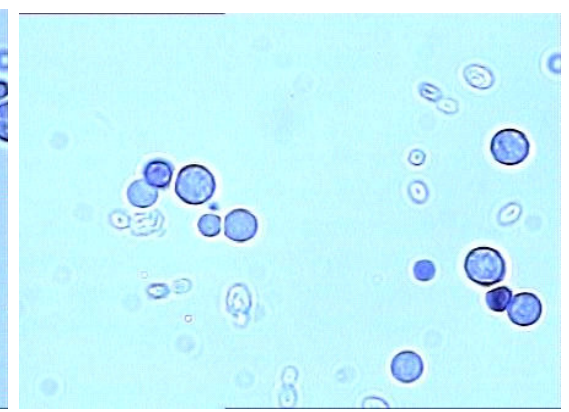

B

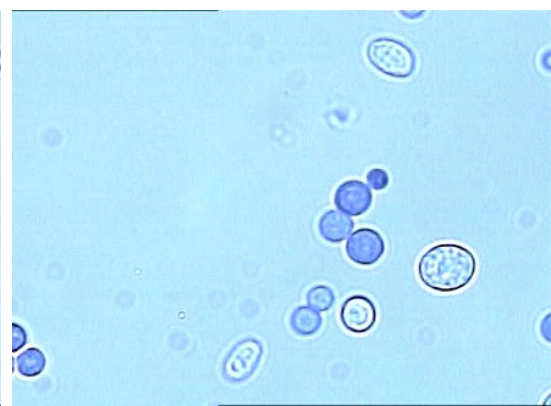

D

Figure 3. Stability of Wan's staining reagent (40x objective). A mixture of Cryptococcus and Candida cells were treated simultaneously with Wan's reagent stored at room temperature for various lengths of time: 1 year $(A), 6$ months $(B), 1$ month $(\mathrm{C})$, and 0 days (i.e., made fresh the day of testing, $D$ ). Wan's reagent proved to be very stable as evidenced by Cryptococcus cells being stained with 1year-old reagent $(A)$, while Candida cells remained unstained.
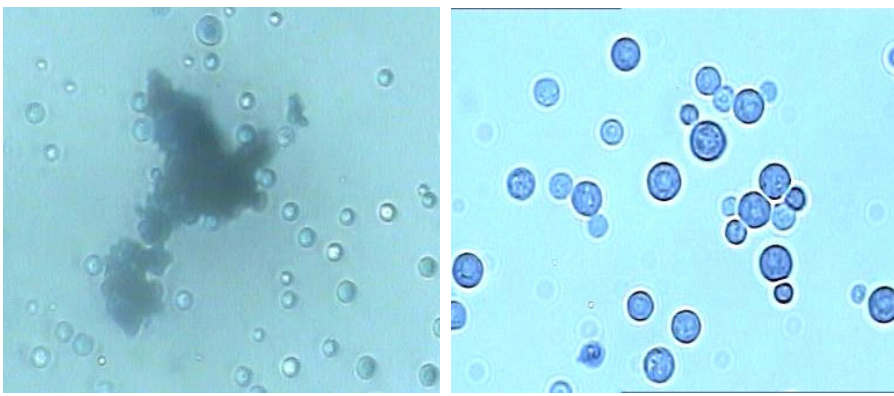

A
B

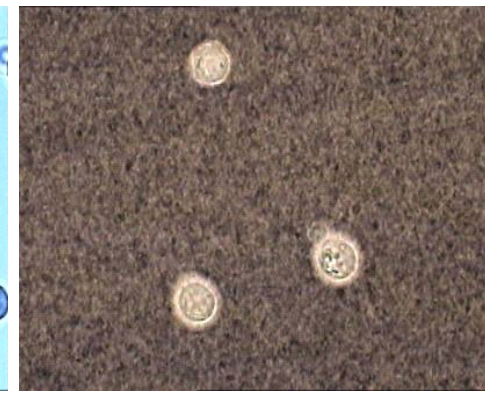

C

Figure 4. Comparison of staining results of three stains on Cryptococcus cells. A: Alcian blue stain: aggregation of Cryptococcal cells often occurred. B: Wan's stain: Cryptococcal cells stained with Wan's reagent appeared blue with bright capsules on a clear background without any aggregation. C: India ink stain: a high level of background staining was observed.

\section{Staining effects of Wan's reagent for cryptococcal cells at high magnification}

We further compared the staining effects of Wan's reagent and India ink staining for cryptococcal cells at high magnification. The high power microscopic appearance [oil immersion (100x) objective] of stained cryptococcal cells demonstrated that Wan's staining 


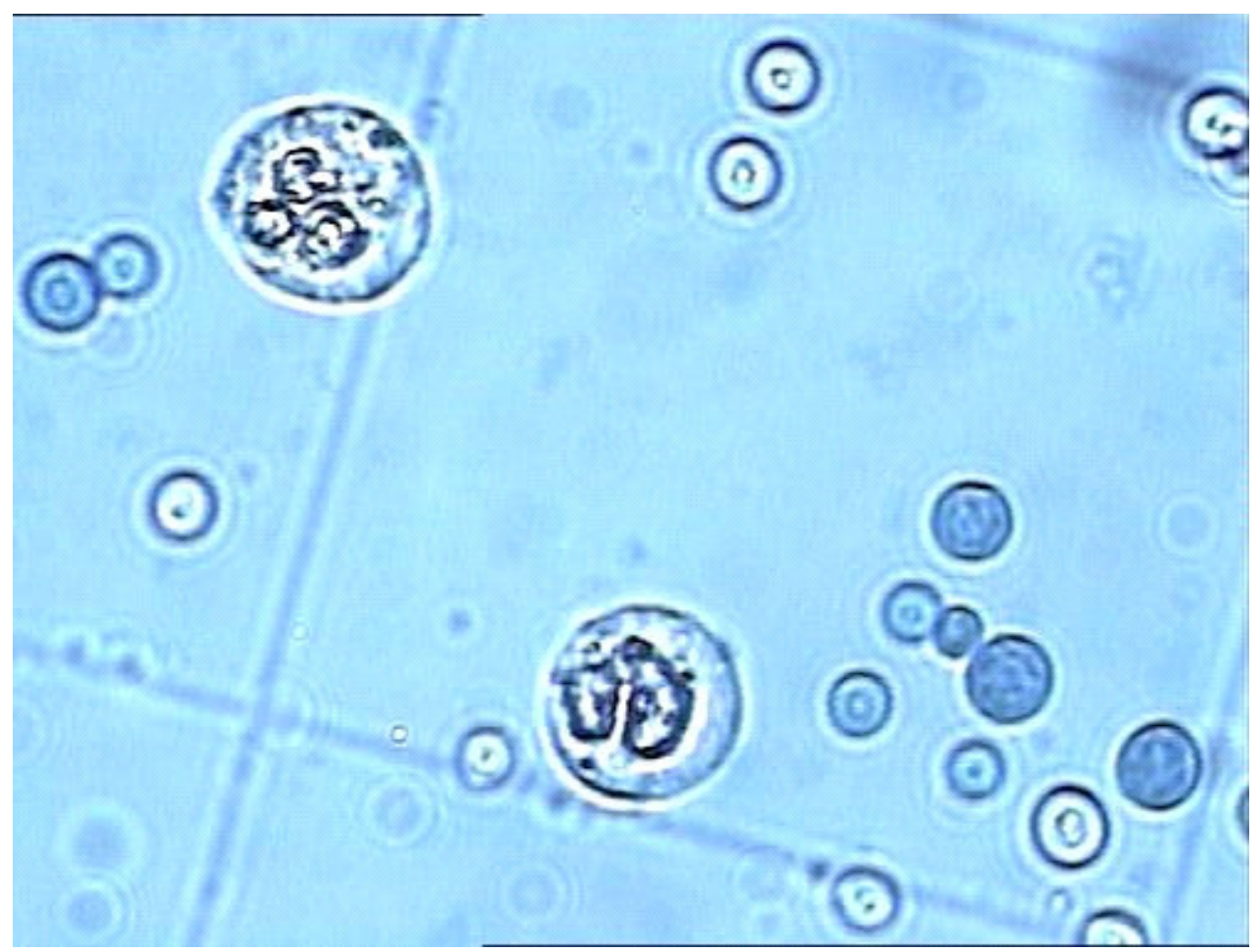

Figure 5. Cryptococcal cells were stained with Wan's reagent and then counted. Cryptococcal cells (blue) were easily distinguishable from Candida cells (white) and white blood cells (relatively bigger size) in the counting chamber $(40 \times$ objective).

reagent was consistently superior to India ink for staining cryptococcal cells, even when examined at high magnification (compare Figure 6A vs. 6B). The intracellular structures of cryptococcal cells stained with Wan's reagent were clearly visible as bright capsules against a clear background (Figure 6B). India ink staining tended to mask cryptococcal cells, particularly small cells, resulting in false negative detections. In contrast, cells stained with Wan's reagent were clearly visible, regardless of size (compare Figure 6C vs. 6D). Thus, Wan's staining enhanced the positive detection rate.

\section{DISCUSSION}

In the present study, we report the testing of Wan's staining reagents, a new staining technique that enables direct counting of Cryptococcus cells under a microscope and which may replace traditional India ink staining for identification of Cryptococcus. Our data demonstrated that Cryptococcus cell membranes were stained dark blue, while cell bodies were stained light blue. Our novel stain stains cryptococcal cells with high specificity as evidenced by the observation that other "yeast-like" fungi and white blood cells were not stained, with the exception of a small portion of $C$. rugosa cells. However, this very weak staining of $C$. rugosa fungi by Wan's reagent could be completely prevented by increasing the $\mathrm{pH}$ value of the reagent with no effect on Cryptococcus staining (data not shown).

It is noteworthy that Wan's staining reagent is very stable. Cryptococcal cells were still stained well with 1year-old Wan's reagent. Furthermore, Wan's staining reagent can be conveniently used to stain Cryptococcus for $1 \mathrm{~min}$ to $1 \mathrm{~h}$ with similar excellent results. Our observations indicate that this novel staining technique is a better alternative approach than India ink or Alcian blue staining for identifying Cryptococcus. Wan's reagent increased the positive detection rate, particular for junior laboratory technicians generally lacking experience. As mentioned earlier, staining and counting of Cryptococcus cells with this new technique could be accomplished in only a few minutes, and this temporal advantage of Wan's reagent is important for its potential clinical application as a rapid diagnostic method for Cryptococcus infections. Because Wan's stain allowed for clear visualization of intracellular structures, unlike India ink staining, Wan's reagent may also benefit other researchers studying the morphology of Cryptococcus.

The Cryptococcus cell wall contains melanin, which may affect its porosity (Gomez and Nosanchuk, 2003; Jacobson and Ikeda, 2005); high porosity allows charged small-molecular dyes to permeate into cryptococcal cells. The staining mechanism of Wan's reagent may be 
Table 1. Comparison of cell count precision between Wan's stain and Alcian blue stain for cryptococcal cells at three different concentrations (30 tests per condition).

\begin{tabular}{|c|c|c|c|c|c|c|}
\hline \multirow{2}{*}{ Stain } & \multicolumn{2}{|c|}{ High concentration $2 \times 10^{3}$ cells $/ \mu \mathrm{l}$} & \multicolumn{2}{|c|}{ Medium concentration $2 \times 10^{2}$ cells $/ \mu \mathrm{l}$} & \multicolumn{2}{|c|}{ Low concentration $2 \times 10$ cells $/ \mu \mathrm{l}$} \\
\hline & $\bar{x} \pm s$ (cells/ $/ \mu \mathrm{l})$ & CV (\%) & 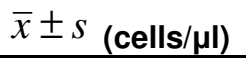 & CV (\%) & $\bar{x} \pm s($ cells $/ \mu \mathrm{l})$ & CV (\%) \\
\hline Wan's & $2061.5 \pm 50.2$ & 2.4 & $248.2 \pm 17.5$ & 7.0 & $22.6 \pm 2.6$ & 11.5 \\
\hline Alcian blue & $1313.2 \pm 246.8$ & 18.8 & $96.3 \pm 23.6$ & 24.5 & $18.4 \pm 2.3$ & 12.5 \\
\hline
\end{tabular}

Each result is the mean of three observations. CV: coefficient of variation.

$\mathbf{A}$
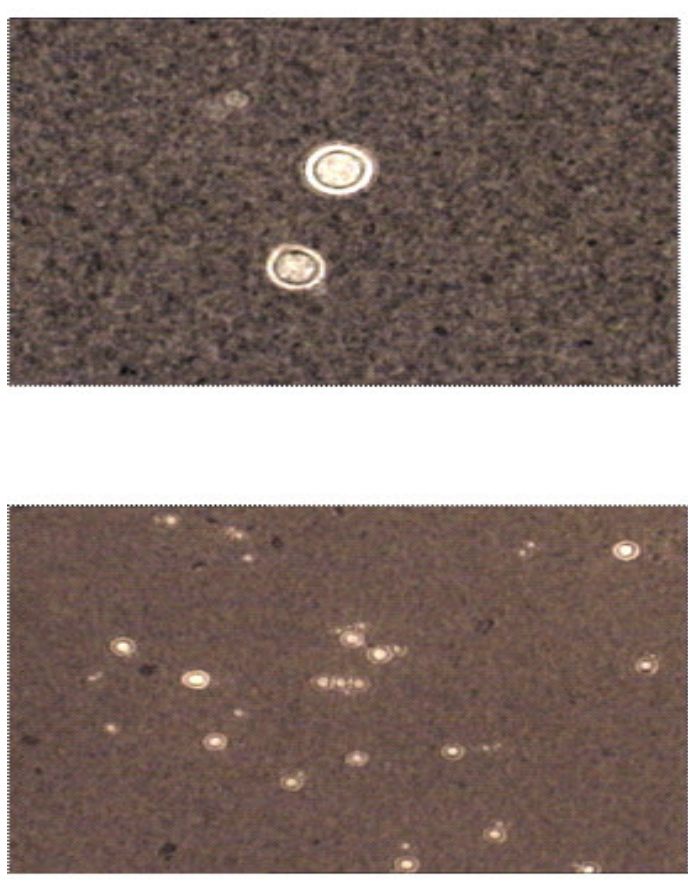

C
B
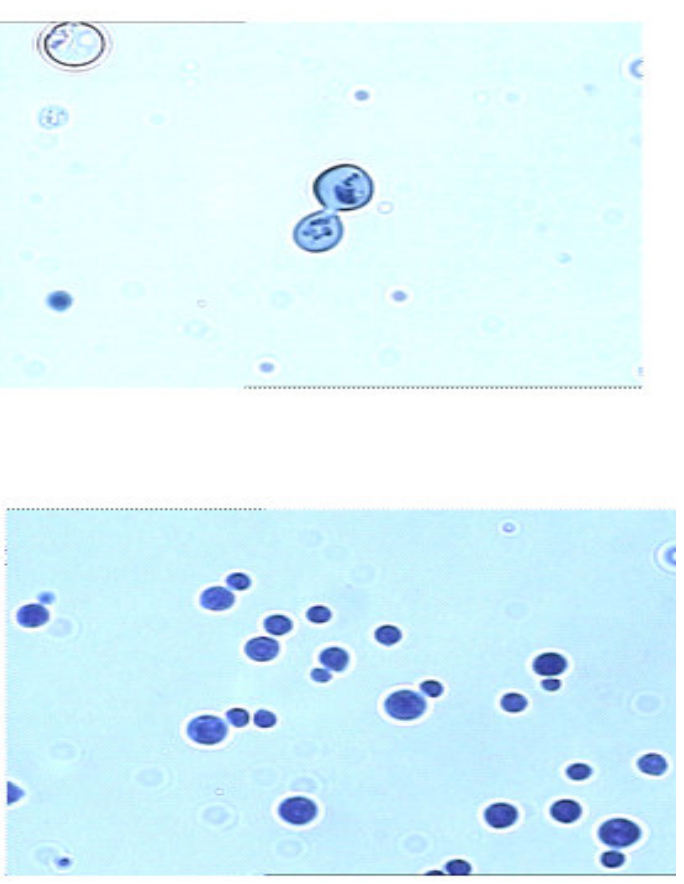

$\mathbf{D}$

Figure 6. Comparison of Wan's reagent and India ink staining of Cryptococcus cells. A: India ink-stained Cryptococcus cells with bright capsules $(100 \times$ objective). B: The intracellular structures of Wan's-stained cryptococcal cells are clearly visible with bright capsules, a clear background and darkly stained envelopes (100x objective). C: India ink-stained Cryptococcus cells with bright capsules and several fungi cells masked by India ink in the field of vision (40x objective). D: Cryptococcal cells were stained with Wan's reagent, resulting in a clear background. No fungi cells were masked by Wan's reagent. 
related to this permeability, but further studies are needed to elucidate the exact mechanism.

\section{Conclusion}

We developed a new staining technique and directly counted cells with a blood cell counter to rapidly and specifically identify Cryptococcus cells. Detection of Cryptococcus with Wan's reagent can be accomplished with one step, qualitatively and quantitatively, highlighting its potential application in the rapid identification and diagnosis of Cryptococcus infections in clinical settings. In addition, this rapid staining technique only requires simple instruments, such as a bench top light microscope. Thus, Wan's staining reagent is well suited for clinical use, including in small and/or community hospitals in developing countries.

\section{ACKNOWLEDGEMENTS}

This work was supported by a grant from the Medical and Health Research Foundation of Zhejiang Province (number: 2009B162). We thank Huo-Xiang Lu (People's Hospital of Zhejiang Province), Hai-Shen Kong and Qing Yang (Zhejiang University), Chang-Gui Sun (the 117th Affiliated Hospital of PLA) and Yun-Wen Zhao (Clinical Laboratory Center of Zhejiang Province) for their technical assistance and/or providing fungi strains.

\section{REFERENCES}

Baker RD, Haugen RK (1955). Tissue changes and tissue diagnosis in cryptococcosis; a study of 26 cases. Am. J. Clin. Pathol., 25: 14-24.

Berlin L, Pincus JH (1989). Cryptococcal meningitis: false-negative antigen test results and cultures in nonimmunosuppressed patients. Arch. Neurol., 46: 1312-1316.

Bloomfield N, Gordon MA, Elmendorf DF (1963). Detection of Cryptococcus neoformans Antigen in Body Fluids by Latex Particle Agglutination. Proc. Soc. Exp. Biol. Med., 114: 64-67.

Bovers M, Hagen F, Boekhout T (2008). Diversity of the Cryptococcus neoformans-Cryptococcus gattii species complex. Rev. Iberoam. Micol., 25: S4-12.
Butler WT, Alling DW, Spickard A, Utz JP (1964). Diagnostic and Prognostic Value of Clinical and Laboratory Findings in Cryptococcal Meningitis, a Follow-up Study of Forty Patients. N. Engl. J. Med., 270: 59-67.

Chayakulkeeree M, Perfect JR (2006). Cryptococcosis. Infect. Dis. Clin. North Am., 20: 507.

Gal AA, Koss MN, Hawkins J, Evans S, Einstein H (1986). The pathology of pulmonary cryptococcal infections in the acquired immunodeficiency syndrome. Arch. Pathol. Lab. Med., 110: 502-507.

Gazzoni AF, Pegas KL, Severo LC (2008). Histopathological techniques for diagnosing cryptococcosis due to capsule-deficient Cryptococcus: case report. Rev. Soc. Bras. Med. Trop., 41: 76-78.

Gazzoni AF, Severo CB, Barra MB, Severo LC (2009). Atypical micromorphology and uncommon location of cryptococcosis: a histopathologic study using special histochemical techniques (one case report). Mycopathology, 167: 197-202.

Gomez BL, Nosanchuk JD (2003). Melanin and fungi. Curr. Opin. Infect. Dis., 16: 91-96.

Harding SA, Scheld WM, Feldman PS, Sande MA (1979). Pulmonary infection with capsule-deficient Cryptococcus neoformans. Virchows. Arch. A. Pathol. Anat. Histol., 382: 113-118.

Jacobson ES, Ikeda R (2005). Effect of melanization upon porosity of the cryptococcal cell wall. Med. Mycol., 43: 327-333.

Johnson LB, Bradley SF, Kauffman CA (1998). Fungaemia due to Cryptococcus laurentii and a review of non-neoformans cryptococcaemia. Mycoses, 41: 277-280.

Kamalam A, Yesudian P, Thambiah AS (1977). Cutaneous infection by Cryptococcus laurentii. Br. J. Dermatol., 97: 221-223.

Kordossis T, Avlami A, Velegraki A, Stefanou I, Georgakopoulos G, Papalambrou C, Legakis NJ (1998). First report of Cryptococcus laurentii meningitis and a fatal case of Cryptococcus albidus cryptococcaemia in AIDS patients. Med. Mycol., 36: 335-339.

Lazcano O, Speights VO Jr, Strickler JG, Bilbao JE, Becker J, Diaz J (1993). Combined histochemical stains in the differential diagnosis of Cryptococcus neoformans. Mod. Pathol., 6: 80-84.

Leal AL, Faganello J, Fuentefria AM, Boldo JT, Bassanesi MC, Vainstein MH (2008). Epidemiological profile of cryptococcal meningitis patients in Rio Grande do Sul, Brazil. Mycopathologia, 166: $71-75$.

McCurdy LH, Morrow JD (2001). Ventriculitis due to Cryptococcus uniguttulatus. South. Med. J., 94: 65-66.

Monteil RA, Hofman P, Michiels JF, Loubiere R (1997). Oral cryptococcosis: Case report of salivary gland involvement in an AIDS patient. J. Oral. Pathol. Med., 26: 53-56.

Perfect JR, Casadevall A (2002). Cryptococcosis. Infect. Dis. Clin. North. Am., 16: 837-874.

Ritterband DC, Seedor JA, Shah MK, Waheed S, Schorr I (1998). A unique case of Cryptococcus laurentii keratitis spread by a rigid gas permeable contact lens in a patient with onychomycosis. Cornea, 17: 115-118.

Velez A, Fernandez-Roldan JC, Linares M, Casal M (1996). Melanonychia due to Candida humicola. Br. J. Dermatol., 134: 375376. 